\title{
The effect of a secretion-enhanced heavy chain on improving intein-based dual-vector co-delivery of a full-length factor VIII gene
}

\author{
ZHU FuXiang*, YANG ShuDe, LIU ZeLong, MIAO Jing, QU HuiGe \& CHI XiaoYan \\ College of Life Sciences, Ludong University, Yantai 264025, China
}

Received July 13, 2010; accepted October 22, 2010

\begin{abstract}
Treatment of hemophilia A by gene therapy is adversely affected by inefficient FVIII secretion and the large FVIII gene, which is difficult to package in the promising adeno-associated virus (AAV) vectors. Inhibited secretion of FVIII is caused mainly by inefficient secretion of its heavy chain. Previously, we have employed a protein splicing-based dual-vector to co-transfer a B-domain-deleted FVIII (BDD-FVIII) gene, suggesting that the light chain, covalently ligated to a co-expressed heavy chain can improve the secretion of spliced BDD-FVIII. However, its level of secretion was affected by inefficient secretion the heavy chain. Here, we studied the effect of a mutant heavy chain with L303E/F309S substitutions, which enhance FVIII secretion on the heavy chain itself and spliced FVIII when using a protein splicing-based split-delivery of a full-length FVIII gene. Eukaryotic vectors expressing Ssp DnaB intein-fused mutant heavy and light chains were transiently co-transfected into cultured COS-7 cells. A spliced FVIII protein was seen in co-transfected cells by Western blot analysis. The heavy chain was secreted by cells transfected with the mutant heavy chain gene alone at $(39 \pm 11) \mathrm{ng} / \mathrm{mL}$ and this secretion increased to $(123 \pm 13) \mathrm{ng} / \mathrm{mL}$ when cells were co-transfected with the light chain gene, which was greater than the secretion of wild-type heavy chain. The amount of spliced FVIII in the culture supernatant of co-transfected cells was $(86 \pm 14) \mathrm{ng} / \mathrm{mL}$, with an activity of $(0.61 \pm 0.08) \mathrm{IU} / \mathrm{mL}$, which was greater than that of wild-type FVIII co-transfected cells. Spliced FVIII and bioactivity were also detected in the combined culture supernatant of cells individually transfected with mutant heavy and light chain gene at a higher level than that of combined wild-type heavy and light chain transfections. This suggested that the heavy chain with improved secretion markedly increased the efficacy of protein splicing-based split delivery of the full-length FVIII gene using a dual-vector. These results encourage the transfer of this technology to an animal model using a dual-AAV vector.
\end{abstract}

coagulation factor VIII, secretion, mutant heavy chain, intein, protein splicing

Citation: Zhu F X, Yang S D, Liu Z L, et al. The effect of a secretion-enhanced heavy chain on improving intein-based dual-vector co-delivery of a full-length factor VIII gene. Chinese Sci Bull, 2011, 56: 158-163, doi: 10.1007/s11434-010-4244-7

Coagulation factor VIII (FVIII) is involved in the activation of the intrinsic coagulation pathway, functioning as a cofactor of active FIX to accelerate FX activation. FVIII defects cause hemophilia A, an X-linked bleeding disorder that accounts for the majority of all hemophilia patients [1]. In comparison to current treatment of mainly plasmaderived and recombinant FVIII protein replacement, which is expensive and has a risk of blood-borne virus infection, gene therapy has the potential to provide a cure for hemophilia A [2]. However, because of inefficient secretion of

*Corresponding author (email: fuxiangmail@163.com)
FVIII and the large FVIII gene, which is difficult to package in the promising adeno-associated virus (AAV) vectors, progress of hemophilia A gene therapy has been slow.

FVIII binds to immunoglobulin-binding protein (BiP), an endoplasmic reticulum (ER) chaperone protein, during secretion and the release of FVIII is dependent on a high concentration of intracellular ATP. L303E/F309S mutations in the A1 domain of the FVIII heavy chain improve its secretion by reducing the affinity of FVIII for BiP $[3,4]$. As an alternative technology, dual-vector co-delivery of FVIII heavy and light chain genes can overcome the AAV capacity constraints, but its main drawback is chain imbalance 
due to the low secretion of the heavy chain relative to efficient light chain secretion [5,6]. An intein is a protein sequence embedded in-frame within a precursor protein sequence that is excised during the maturation process of protein splicing, which is an analog of RNA splicing [7]. Protein splicing has been applied in protein engineering and other fields in recent years as a technical tool. We have recently used a protein splicing-based dual-vector system for co-delivery of a B-domain-deleted FVIII (BDD-FVIII) gene and demonstrated that the co-expressed heavy and light chains can be spliced to form a complete BDD-FVIII, and that the light chain shows a cis-promoting function for the secretion of spliced BDD-FVIII [8,9]. Surprisingly, the secretion of L303E/F309S BDD-FVIII heavy chain by itself was even lower than its wild-type form and BDD-FVIII [8]. In the current study, we utilized a protein splicing-based dual-vector to co-transfer the full-length FVIII gene with L303E/F309S mutations to investigate the secretion of the mutant heavy chain and spliced FVIII. The data showed an obviously improved secretion of the mutant heavy chain by itself and spliced FVIII with an increased bioactivity. This provides an experimental basis for transferring dual-AAV vector co-delivery of full-length FVIII gene to animal models.

\section{Materials and methods}

\subsection{Materials}

pXX-FVIII, containing a full-length human FVIII cDNA, was constructed and kindly provided by Dr. Xiao Xiao of Pittsburg University. The expression vectors for $S s p$ DnaB intein-infused FVIII heavy chain with F309S mutation, pCMV-HIn, for Ssp DnaB intein-infused FVIII light chain, pCMV-IcL and for full-length FVIII, pCMV-FVIII were previously constructed in our laboratory. COS-7 cells were obtained from the Chinese Academy of Sciences.

The high fidelity DNA polymerase Pfu Turbo was obtained from Stratagene (Santa Clara, CA, USA). The Gel Extraction kit, PCR purification kit and Spin Miniprep kit were obtained from Qiagen (Germany). The cell culture and transfection reagents were obtained from Invitrogen. The fetal bovine serum was from Hyclone (South Logan, UT, USA). The recombinant human factor VIII was from BioChain (Hayward, CA, USA). The FVIII heavy chain-specific monoclonal antibody, ESH5, light chain-specific monoclonal antibody, ESH8, and the horseradish peroxidase (HRP)-conjugated ESH8, HRP-ESH8, were purchased from American Diagnostica (Stamford, CT, USA); HRP-conjugated rabbit anti-human FVIII polyclonal antibody was purchased from Novus (Littleton, CO, USA); HRP-conjugated sheep anti-rabbit secondary antibody and the ECL Plus Western blotting detection kit were obtained from Amersham Pharmacia Biotech (Piscataway, NJ, USA). Normal human reference plasma was obtained from George King Biomedical (Overland Park, KA, USA). A chromogenic kit for the determination of FVIII activity, COATEST SP FVIII was purchased from Chromogenix (Milan, Italy).

\subsection{Vector construction}

The intein-fused L303E/F309S heavy chain expression vector, pCMV-MHCIn, was constructed by introducing the L303E mutation into the FVIII heavy chain by inverse PCR using Pfu Turbo DNA polymerase, primers (5'-AGGACAGTTTCTACTGTTTTG-3' and 5'-TCGTCCATCAAGAGTGTTTG-3') with pCMV-HIn, containing Ssp DnaB intein-fused F309S heavy chain, as a template followed by self-ligation of the PCR product. To construct the Ssp DnaB intein-fused wild-type heavy chain expression vector, pCMV-HCIn, inverse PCR was carried out using primers (5'-CTTGTCATATCTCTTCCCACCA-3' and 5'-ACAGTAGAAACTGTCCAAGGT-3') with pCMV-HIn as template to convert the Glu330 codon to Leu followed by circularization of the PCR product. For convenience, the plasmid pCMV-IcL, expressing Ssp DnaB intein-fused light chain constructed previously, was renamed as pCMV-IcLC.

\subsection{Cell culture and gene transfection}

COS-7 cells were maintained at $37^{\circ} \mathrm{C}$ in an atmosphere of $5 \% \mathrm{CO}_{2}$ and were grown in Dulbecco's modified Eagle's medium (DMEM) supplemented with $10 \%$ fetal bovine serum. $24 \mathrm{~h}$ before transfection, cells were harvested using $0.05 \%$ trypsin and plated onto a 6 -well plate at $5 \times 10^{5}$ cells per well. When the cells were $90 \%$ confluent, they were co-transfected with the pCMV-MHCIn and pCMV-IcLC plasmids using Lipofectamine 2000 (Invitrogen) in accordance with the manufacturer's instructions. $6 \mu \mathrm{g}$ of each plasmid was diluted in $250 \mu \mathrm{L}$ of Opti-MEM and gently mixed with $20 \mu \mathrm{L}$ of lipofectin in $250 \mu \mathrm{L}$ of Opti-MEM for $5 \mathrm{~min}$ at room temperature, followed by further $20 \mathrm{~min}$ incubation at room temperature without shaking, before adding the complexes to the cells. As a control, cells were co-transfected with pCMV-HCIn and pCMV-IcLC. In addition, pcDNA3.1 and pCMV-FVIII were transfected into cells as a mock control and to express full-length FVIII, respectively. After incubation at $37^{\circ} \mathrm{C}$ in a $\mathrm{CO}_{2}$ incubator for $5 \mathrm{~h}$, the culture media was changed to fresh Opti-MEM and the cells were incubated for another $48 \mathrm{~h}$ prior to testing transgene expression and protein trans-splicing. To observe protein splicing after secretion, cells were separately transfected with pCMV-MHCIn (or pCMV-HCIn) and pCMV-IcLC, then combined together followed by incubation for $24 \mathrm{~h}$. The culture supernatants were collected for analysis.

\subsection{Western blot analysis for FVIII splicing}

For Western blot analysis, cells were lysed by repeated freeze-thawing, in liquid nitrogen and a water bath at $37^{\circ} \mathrm{C}$. After protein quantification by the Bradford method, $12 \mu \mathrm{g}$ 
of total cellular proteins were resolved and separated by SDS-PAGE and transferred onto PVDF membrane by a semi-dry method. After blocking the membrane with 5\% skimmed milk powder at room temperature for $2 \mathrm{~h}$, it was incubated with a rabbit anti-human FVIII polyclonal antibody for $1 \mathrm{~h}$ at $37^{\circ} \mathrm{C}$ followed by HRP-conjugated secondary antibody for $1 \mathrm{~h}$ at $37^{\circ} \mathrm{C}$. Hybridization was visualized with the ECL Plus Western blotting detection system.

\subsection{Determination of secreted heavy and light chains and spliced FVIII}

The concentration of heavy and light chains in the culture supernatants was measured by heavy chain and light chainspecific ELISA, respectively, as described previously [8].

A sandwich ELISA was performed to measure the concentration of the spliced full-length FVIII protein in the culture supernatant using chain-specific antibodies [10]. The FVIII heavy and light chain antibodies were used as capture and detection antibodies. NUNC Maxisorb 96-well plates were coated overnight at $4^{\circ} \mathrm{C}$ with $2 \mu \mathrm{g} / \mathrm{mL}$ FVIII heavy chain-specific monoclonal antibody ESH5. The plates were washed with phosphate-buffered saline (PBS)-0.05\% Tween 20 and then blocked with PBS-1\% bovine serum albumin (BSA)- $0.05 \%$ Tween 20 for $2 \mathrm{~h}$ at $37^{\circ} \mathrm{C} .100 \mu \mathrm{L}$ of standard recombinant FVIII dilutions in Opti-MEM, ranging in concentration from 10 to $300 \mathrm{ng} / \mathrm{mL}$, and samples were added to wells, in triplicate. The plates were incubated for $1 \mathrm{~h}$ at $37^{\circ} \mathrm{C}$ and washed 3 times. $100 \mu \mathrm{L}$ of HRP-conjugated light chainspecific antibody, HRP- ESH8, at a concentration of $2 \mu \mathrm{g} / \mathrm{mL}$ was added to each well and the plates were incubated for $1 \mathrm{~h}$ at $37^{\circ} \mathrm{C}$. After a final wash, the substrate OPD was added and the plates were incubated for $30 \mathrm{~min}$ at $37^{\circ} \mathrm{C}$. The reaction was terminated with $2 \mathrm{~mol} / \mathrm{L} \mathrm{H}_{2} \mathrm{SO}_{4}$, then the absorbance was read at $490 \mathrm{~nm}$. A standard working curve was plotted based on the absorbance versus concentration of the diluted standards, and FVIII concentration in the supernatant was determined from the standard curve.

\subsection{Analysis of FVIII bioactivity of culture super- natants}

The activity produced by FVIII in the supernatant was measured using the Coatest chromogenic method [11] in accordance with the manufacturer's instructions. Normal human plasma was used as a standard with a defined FVIII activity of $1.0 \mathrm{IU} / \mathrm{mL}$. A standard curve was generated from $405 \mathrm{~nm}$ absorbance versus FVIII activities. The absorbance of samples was also read at $405 \mathrm{~nm}$ after serial reactions and the FVIII activity was deduced from the standard curve.

\subsection{Statistical analysis}

Data were expressed as mean \pm SD. Significant differences among the groups were determined using Student's paired $t$ test and a value of $P<0.05$ was considered significant.

\section{Results}

\subsection{Construction of intein-fused L303E/F309S FVIII heavy chain expression vector}

The structural domains of the full-length FVIII molecule are arranged as A1-A2-B-A3-C1-C2. Intein-fused wild-type heavy and light chain gene expression vectors, pCMV-HCIn and pCMV-IcLC were previously constructed by splitting the FVIII gene between Gly1238 and Ser1239 in the B domain into heavy and light chains, fusing $S s p$ DnaB intein Nand C-terminus, to them respectively, followed by insertion into the eukaryotic expression vector pcDNA3.1(+). The intein-fused L303E/F309S heavy chain expression vector pCMV-MHCIn was produced by introducing the L303E mutation into the intein-fused F309S heavy chain expression vector, pCMV-HIn, by PCR-directed mutagenesis. The structures of FVIII and of the fusion gene expression vectors are illustrated in Figure 1.

\subsection{Intracellular splicing of FVIII protein}

Western blot of total cellular protein showed that the FVIII transgenic cells display an expressed FVIII protein of the expected size but that the mock transfected cells have no visible FVIII protein. The MHCIn and IcLC co-transfected cells displayed an obviously spliced FVIII (MHC-LC) consistent in size with FVIII protein. They also displayed nonspliced protein precursors; intein-fused heavy and light chains (MHCIn and IcLC) were also detected, in a similar manner to that in cells separately transfected with $\mathrm{MHCIn}$ and $I C L C$ genes. There may be two reasons for the noncomplete splicing, one is the protein splicing reaction itself not entirely efficient and the other is the low efficiency of both intein-fused genes entering into the same target cell so that the expressed precursor proteins cannot meet intracellularly for splicing (Figure 2).

\subsection{Improved heavy chain secretion and its role in promoting secretion of spliced FVIII}

Heavy chain-specific ELISA showed that the concentration of mutant heavy chain in the culture supernatant secreted by cells transfected with the MHCIn gene alone is $(39 \pm 11)$ $\mathrm{ng} / \mathrm{mL}$, which was significantly higher than wild-type heavy chain secreted from HCIn transgenic cells $(15 \pm 5 \mathrm{ng} / \mathrm{mL})$, suggesting improvement of mutant heavy chain secretion. After co-transfection with the IcLC gene, secretion of the mutant heavy chain markedly increased to $(123 \pm 13) \mathrm{ng} / \mathrm{mL}$, which is composed of spliced FVIII and unspliced heavy chain, indicating the cis-promoting effect of the light chain on the secretion of mutant heavy chain. This was better than 
FVIII

\begin{tabular}{|l|l|l|l|l|l|l|}
\hline SS & A1 & A2 & B & A3 & C1 & C2 \\
\hline
\end{tabular}
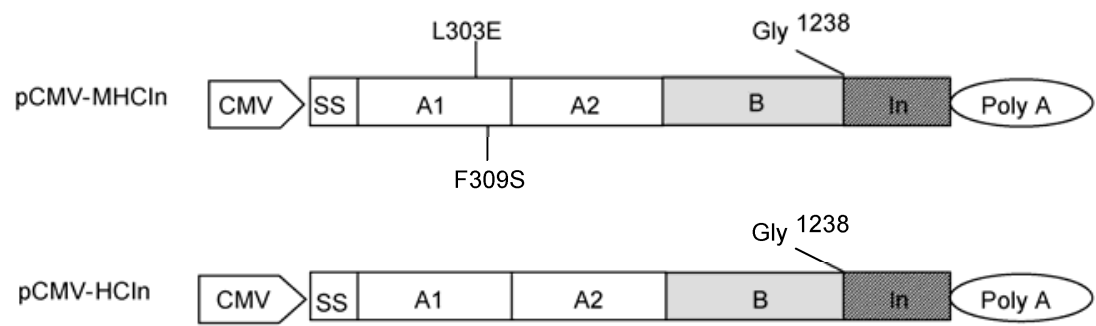

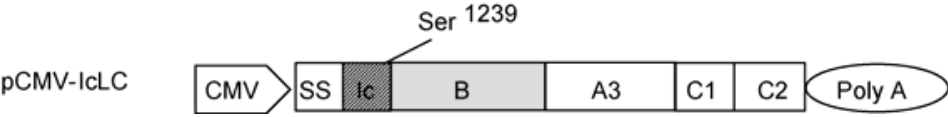

Figure 1 Schematic illustration of FVIII and of the eukaryotic expression plasmid vectors. The structural domains of the full-length FVIII are arranged as A1-A2-B-A3-C1-C2. Intein-joined wild-type heavy chain and light chain expression vectors, pCMV-HCIn and pCMV-IcLC, were constructed by fusing Nand C-terminus (In, Ic) of $S s p$ DnaB intein to heavy and light chains, respectively. The intein-fused L303E/F309S heavy chain expression vector, pCMV-MHCIn, was made by PCR-directed mutagenesis. SS, signal sequence of FVIII.

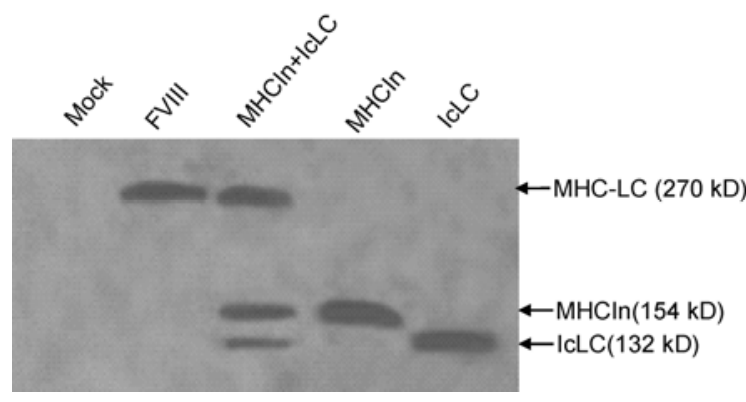

Figure 2 Intracellularly trans-spliced FVIII detected by Western blot. Total proteins in cell lysate were resolved by SDS-PAGE and probed with FVIII polyclone antibody. The MHCIn and ICLC co-transgenic cell shows a spliced FVIII protein (MHC-LC), and also non-completely spliced precursors, MHCIn and IcLC.

the effect on the wild-type heavy chain, which was $(82 \pm 16)$ $\mathrm{ng} / \mathrm{mL}$. The amount of heavy chain secreted by FVIII gene transfected control cells was $(70 \pm 16) \mathrm{ng} / \mathrm{mL}$, which was significantly less than co-transfected cells (Figure 3). Compared to the improved secretion of the mutant FVIII heavy chain, we have recently reported that the secretion of mutated BDD-FVIII heavy chain was even lower than that of BDD-FVIII itself [8].

As shown in Figure 4, a light chain-specific ELISA showed that the light chain secreted from MHCIn and IcLC co-transgenic cells was $(168 \pm 23) \mathrm{ng} / \mathrm{mL}$, which was 1.4 times that of the heavy chain. The light chain secreted from HCIn and IcLC co-transgenic cells was $(189 \pm 29) \mathrm{ng} / \mathrm{mL}$, which was 2.3-fold higher than that of the heavy chain, suggesting that mutant heavy chain and light chain co-transfected cells may alleviate the chain imbalance. The IcLC transgenic cells secreted $(172 \pm 35) \mathrm{ng} / \mathrm{mL}$ of light chain, similar to levels of wild-type or mutant heavy chain plus light chain co-transfected cells, indicating that the heavy chain does not affect the secretion of light chain. These levels were signifi- cantly higher than those of FVIII transgenic control cells $(87 \pm 20 \mathrm{ng} / \mathrm{mL})$ suggesting that secretion of light chain within the FVIII molecule was adversely affected by the inefficient heavy chain secretion (Figure 4).

An intact FVIII molecule could be formed from the synthesized intein-fusion heavy and light chains by protein splicing followed by secretion to the outside of the cell. The unspliced precursor peptides can also be secreted. The amount of spliced FVIII in the culture supernatant determined by sandwich ELISA demonstrated that MHCIn and IcLC in co-transgenic cells was $(86 \pm 14) \mathrm{ng} / \mathrm{mL}$, which was greater than that in HCIn and IcLC co-transgenic cells $(57 \pm 8 \mathrm{ng} / \mathrm{mL})$, and slightly higher than that in FVIII transgenic cells $(75 \pm 12 \mathrm{ng} / \mathrm{mL})$. This indicated that mutation of the heavy chain can improve protein splicing-based dualvector co-delivery of the FVIII gene (Figure 5). A spliced FVIII was also detected at $(23 \pm 5) \mathrm{ng} / \mathrm{mL}$ in the culture supernatant of combined cells individually transfected with MHCIn and ICLC genes, which was at a higher level than that of combined HCIn and IcLC transgenic cells $(13 \pm 4$ $\mathrm{ng} / \mathrm{mL}$ ) implying cellular mechanism-independent protein splicing and that the improved heavy chain secretion was beneficial to this kind of splicing.

\subsection{FVIII bioactivity of the culture supernatant}

The FVIII bioactivity of the culture supernatant, produced by spliced FVIII protein, was $(0.61 \pm 0.08) \mathrm{IU} / \mathrm{mL}$ for MHCIn and IcLC co-transgenic cells, which was greater than that of HCIn and IcLC transgenic cells $(0.42 \pm 0.07$ $\mathrm{IU} / \mathrm{mL})$. The bioactivity of culture supernatant of FVIII transgenic control cells was $(0.48 \pm 0.11) \mathrm{IU} / \mathrm{mL}$, which was lower than that of MHCIn and IcLC co-transgenic cells (Figure 6). This suggests that the mutant heavy chain could increase the efficacy of a dual-vector co-delivery of the 


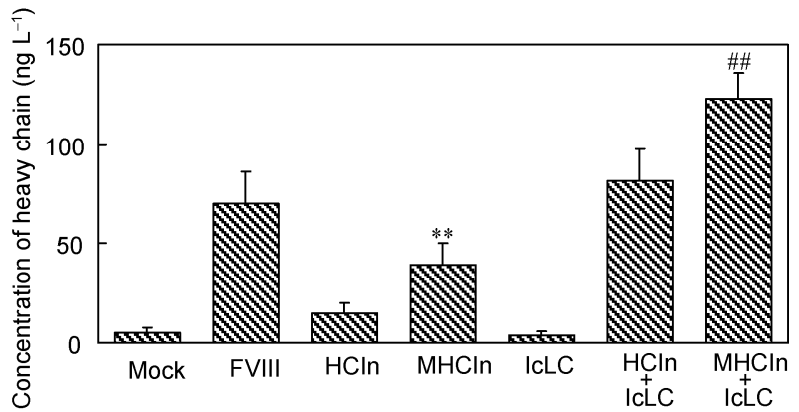

Figure 3 Heavy chain concentration in culture supernatants. **, $P<0.05$ vs. HCIn transfection; \#\#, P<0.05 vs. HCIn and IcLC co-transfection.

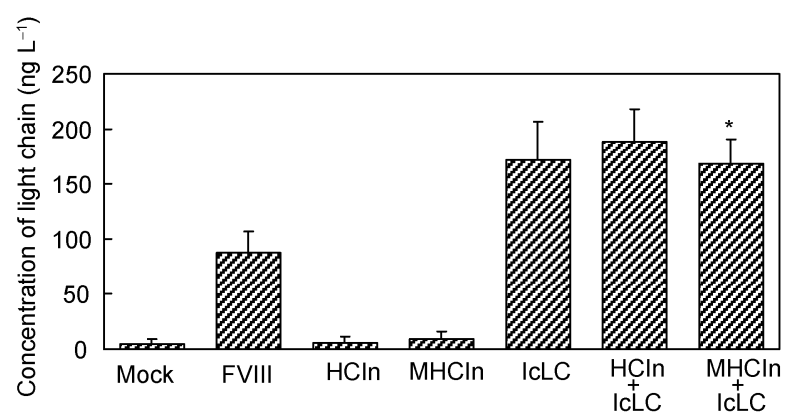

Figure 4 Light chain concentration in culture supernatants. *, $P>0.05$ vs. $H C I n$ plus $I c L C$ co-transfection, and $I c L C$ transfection alone.

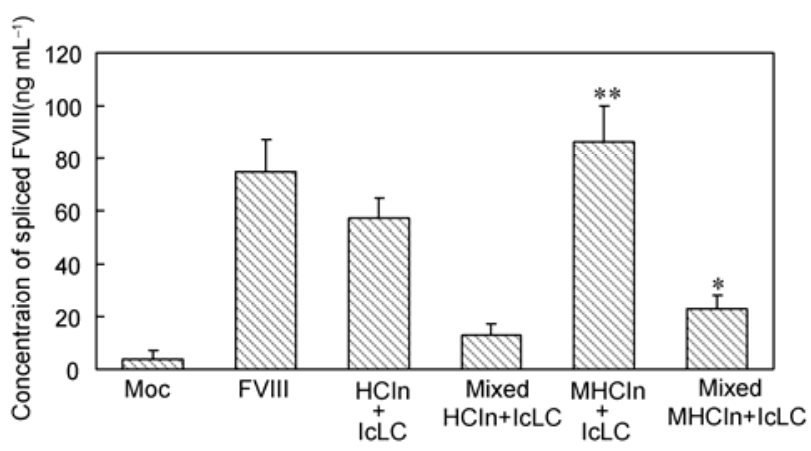

Figure 5 Concentration of spliced FVIII protein in culture supernatants. **, $P<0.05$ vs. HCIn and IcLC co-transfection; *, P>0.05 vs. mixed HCIn and $I c L C$ transfection.

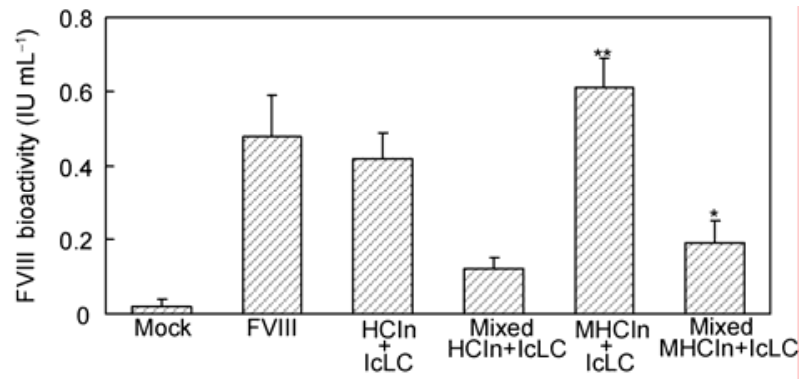

Figure 6 FVIII bioactivity of the culture supernatants. **, $P<0.05$ vs $H C I n+I c L C$ co-transfection; * $P>0.05$ vs. mixed HCIn and IcLC transfection.

FVIII gene. FVIII bioactivity was also detected in culture supernatant of combined cells separately transfected with
MHCIn and $I c L C$ genes $((0.19 \pm 0.06) \mathrm{IU} / \mathrm{mL})$, which was higher than that of combined HCIn and IcLC transgenic cells $((0.12 \pm 0.03) \mathrm{IU} / \mathrm{mL})$, indicating the contribution of cellular mechanism-independent spliced FVIII to bioactivity and that the mutant heavy chain could enhance this function.

\section{Discussion}

Dual-vector co-delivery of the FVIII gene, based on an understanding of physiological FVIII heterodimerization, is an alternative strategy to overcome the limited capacity of AAV vectors in hemophilia A gene therapy $[5,6]$. However, because of a dependence on functional heterodimerization, the transgenic efficacy relies on the efficiency of two vectors entering the same target cells. An additional drawback is the inefficient secretion of heavy chain, which causes chain imbalance with light chain secreted at a rate 10- to 100 -fold higher than heavy chain. The excessively secreted light chain does not, therefore, associate with its counterpart heavy chain and does not contribute to bioactivity $[5,6]$. Furthermore, the heavy chain retained in the cell may destabilize the cell and even induce apoptosis [12].

An interaction between FVIII and the endoplasmic reticulum (ER) chaperone protein $\mathrm{BiP}$ is considered to be the main reason leading to inefficient FVIII secretion [3,1315]. BiP is a peptide-dependent ATPase in the ER and binds unfolded, mutant or unassembled protein subunits within the ER. Release of FVIII bound to BiP during secretion requires high intracellular concentrations of ATP. Depletion of intracellular ATP by treatment with protonophore specifically inhibits secretion of FVIII, whereas secretion of $\mathrm{FV}$, which has a similar structure to FVIII, is not affected $[16,17]$. Amino acid substitution experiments have shown that L303E and F309S point mutations in the A1 domain of the heavy chain reduce the affinity of FVIII with BiP leading to a 3-fold increase in FVIII secretion [4]. We have recently used a dual-vector to transfer a F309S point mutated $B D D-F V I I I$ gene and demonstrated that the light chain could trans-promote secretion of the mutated heavy chain (although only at a level of approximately half that of BDD-FVIII) and that the mutated heavy chain by itself was secreted at even lower levels [8]. Therefore, it is difficult to improve the secretion of BDD-FVIII heavy chain by point mutations using dual-vector technology.

In this study, the full-length FVIII gene with L303E/ F309S mutations in its heavy chain was used for split-delivery by a protein splicing-based dual-vector. The data showed an improved secretion of the mutant heavy chain by itself which increases substantially when co-transfected with the light chain gene with a ratio of heavy to light chain of 1:1.4 compared to $1: 2.3$ in the light and wild-type heavy chain co-transgenic cells. This indicated that the light chain has a more obvious enhancement on mutant heavy chain secretion, which is helpful to relieve the chain imbalance 
problem. Also the amount of spliced FVIII secreted from the cells and its bioactivity were significantly higher compared to that of wild-type FVIII gene co-transgenic cells. Therefore, the covalently ligated FVIII molecules catalyzed by protein splicing in the dual-vector co-delivery of the FVIII gene is similar to a single vector delivery of the full-length FVIII gene.

The protein splicing catalyzed efficient, precise and seamless ligation of proteins, which makes intein a powerful technical tool in protein engineering and drug development $[18,19]$. Recently, by von Willebrand factor (vWF) gene co-delivery, we demonstrated that as a FVIII carrier, the co-expressed vWF is able to promote the secretion of intein spliced BDD-FVIII [20]. This work provided valuable information for the use of protein splicing as a strategy for dual-AAV vector co-delivery of the mutant full-length FVIII gene in animal models to both circumvent the packaging limitation of AAV and to improve the secretion of spliced FVIII.

The authors wish to thank Prof. Paul Liu from the College of Medicine, Dalhousie University, Canada for his support in protein splicing. This work was supported by the Natural Science Foundation of Shandong Province, China (ZR2010CM061), the Science and Technology Program of Yantai (2008152) and the Scientific Research Foundation of Ludong University (LZ20083305).

1 Mann K G. Biochemistry and physiology of blood coagulation. Thromb Haemost, 1999, 82: 165-174

2 Dooriss K L, Denning G, Gangadharan B, et al. Comparison of factor VIII Transgenes bioengineered for improved expression in gene therapy of hemophilia A. Hum Gene Ther, 2009, 20: 465-478

3 Miao H Z, Sirachainan N, Palme L, et al. Bioengineering of coagulation factor VIII for improved secretion. Blood, 2004, 103: 3412-3419

4 Swaroop M, Moussalli M, Pipe S W, et al. Mutagenesis of a potential immunoglobulin-binding protein-binding site enhances secretion of coagulation factor VIII. J Biol Chem, 1997, 272: 24121-24124

5 Burton M, Nakai H, Colosi P, et al. Coexpression of factor VIII heavy and light chain adeno-associated viral vectors produces biologically active protein. Proc Natl Acad Sci USA, 1999, 96: 12725-12730

6 Scallan C D, Liu T, Parker A E, et al. Phenotypic correction of a mouse model of hemophilia A using AAV2 vectors encoding the heavy and light chains of FVIII. Blood, 2003, 102: 3919-3926

7 Perler F B. The ins and outs of gene expression control. Nat Biotechnol, 2004; 22: 824-826

8 Chen L X, Zhu F X, Li J, et al. The enhancing effects of the light chain on heavy chain secretion in split delivery of factor VIII gene. Mol Ther, 2007, 15: 1856-1862

9 Zhu F X, Liu Z L, Chi X Y, et al. Protein trans-splicing based dual-vector delivery of the coagulation factor VIII gene. Sci China Life Sci, 2010, 53: 683-689

10 Gnatenko D V, Saenko E L, Jesty J, et al. Human factor VIII can be packaged and functionally expressed in an adeno-associated virus background: applicability to haemophilia A gene therapy. $\mathrm{Br} \mathrm{J}$ Haematol, 1999, 104: 27-36

11 Sarkar R, Tetreault R, Gao G, et al. Total correction of hemophiliaAmice with canine FVIII using anAAV 8 serotype. Blood, 2004, 103: 1253-1260

12 Zhang K, Shen X, Wu J, et al. Endoplasmic reticulum stress activates cleavage of CREBH to induce a systemic inflammatory response. Cell, 2006, 124: 587-599

13 Kaufman R J, Pipe S W, Tagliavacca L, et al. Biosynthesis, assembly and secretion of coagulation factor VIII. Blood Coagul Fibrinolysis, 1997, 8(Suppl 2): S3-S14

14 Pipe S W. Coagulation factors with improved properties for hemophilia gene therapy. Semin Thromb Hemost, 2004, 30: 227-237

15 Cunningham M A, Pipe S W, Zhang B, et al. LMAN1 is a molecular chaperone for the secretion of coagulation factor VIII. J Thromb Haemost, 2003, 1: 2360-2367

16 Dorner A J, Wasley L C, Kaufman R J. Protein dissociation from GRP78 and secretion are blocked by depletion of cellular ATP levels. Proc Natl Acad Sci USA, 1990, 87: 7429-7432

17 Pittman D D, Tomkinson K N, Kaufman R J. Post-translational requirements for functional factor V and factor VIII secretion in mammalian cells. J Biol Chem, 1994, 269: 17329-17337

18 Gimble F S. Putting protein splicing to work. Chem Biol, 1998, 5: R251-R256

19 Cheriyan M, Perler F B. Protein splicing: A versatile tool for drug discovery. Adv Drug Deliv Rev, 2009, 61: 899-907

20 Zhu F X, Yang S D, Liu Z L, et al. vWF improves secretion and activity of intein spliced BDD-FVIII (in Chinese). Acta Pharm Sin, 2010, 45: 595-600

Open Access This article is distributed under the terms of the Creative Commons Attribution License which permits any use, distribution, and reproduction in any medium, provided the original author(s) and source are credited. 